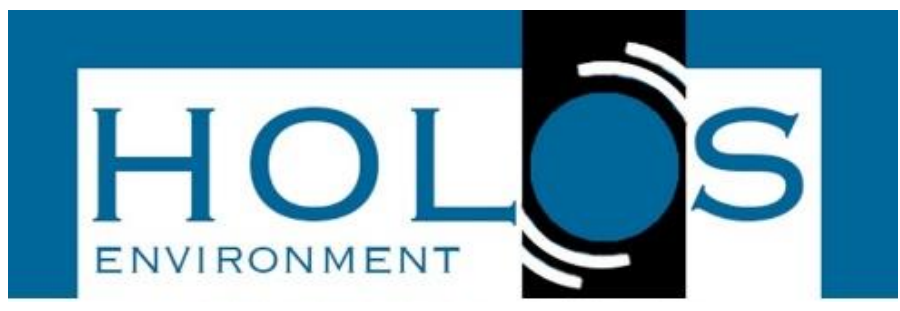

\title{
O ESTADO DA ARTE EM ECOLOGIA DO SOLO
}

\author{
STATE OF THE ART IN SOIL ECOLOGY
}

\author{
Thamyres Sabrina Gonçalves ${ }^{1}$
}

Artigo recebido em: 29/09/2014 e aceito para publicação em: 05/05/2019. DOI: http://dx.doi.org/10.14295/holos.v19i1.9206

Resumo: O ecossistema solo é amplamente divulgado como sendo um dos mais ricos ecossistemas do mundo todavia, apesar de ser reconhecida essa grande complexidade ecossistêmica do solo pouco se conhece acerca das interações que ocorrem nesse ecossistema. As interações que são conhecidas a maior parte delas não foram estudadas dentro da dinâmica natural de funcionamento do ecossistema do solo. Portanto, são amplamente necessários trabalhos que investiguem o estado da arte do conhecimento em ecologia do solo, biologia do solo e de todas as interações que acontecem nesse ecossistema, pois somente a partir do conhecimento e entendimento do que já fora gerado em termos de conhecimento cientifico sobre esses processos, abaixo e acima da camada edáfica superficial é que poderemos avançar de modo a produzir um conhecimento para atender as demandas acerca dos processos ecológicos no solo. Os resultados dessa revisão bibliográfica mostram que houveram importantes avanços na ciência acerca dos processos de interação ecológica no ecossistema solo e que em muito contribuíram para a ecologia, mas que ainda existem muitos temas ou tópicos a serem abordados e organismos pouco ou quase nada estudados. Conclui-se que o ecossistema solo pode ser um ambiente de grandes contribuições para a compreensão de como os processos antrópicos têm influenciado a biodiversidade terrestre e também como poderemos estudar a recuperação dos processos biológicos que foram perdidos em alguns ecossistemas.

Palavras-chave: Biologia do solo. Ecossistema edáfico. Interações multitróficas.

Abstract: The soil ecosystem is widely reported as being one of the richest ecosystems in the world however, despite being recognized this great ecosystemic complexity Soil little is known about the interactions that occur in this ecosystem. The interactions that are known not been studied within the natural dynamics of functioning of the soil ecosystem. So, are widely needed studies that investigate the state of the art knowledge on soil ecology, soil biology and all the interactions that take place in this ecosystem, because only from the knowledge of where we are in terms of scientific knowledge about these processes interaction in the ground, below and above the surface layer soil organisms is that we can move forward in order to produce knowledge that can meet the demands on the ecological processes in soil. The results of this literature review shows that there have been important advances in science about the processes of ecological interactions in the soil ecosystem and contributed greatly to the ecology, but there are still many issues and little or nothing organisms studied. It was concluded that the soil ecosystem can be an environment of great contributions to the understanding of how the anthropogenic processes have influenced the Earth's biodiversity and also how we can do to try to recover the biological processes that have been lost in some ecosystems.

Palavras-chave: Biology of soil. Edaphic ecossystem. Multitrophic interactions.

\footnotetext{
${ }^{1}$ Universidade Federal dos Vales do Jequitinhonha e Mucuri (UFVJM), Teófilo Otoni, MG . E-mail: (sabrina5thamy@yahoo.com.br)
} 


\section{INTRODUÇÃO}

Desde a sua presença na terra o ser humano se preocupa em estudar e conhecer melhor sobre os solos, suas cores, sua fertilidade natural, condições físicas e produtividade agrícola (LEPSCH, 2002). Todavia os processos biológicos e as interações ecológicas não estiveram desde sempre dentre as prioridades no estudo dos solos de acordo com Lepsch (2002).

Apesar disso, Denardin (et al, 2013) em um levantamento histórico do conceito de fertilidade de solo abordou que desde o princípio da civilização do século I ao $X$ a concepção de solo fértil baseava-se na integração das fases biológica, física e química do solo, em contínua renovação pelo cultivo com técnicas apropriadas, ou melhor dizendo, na capacidade natural de resiliência do solo frente aos processos de modificação pelos quais estivesse passado.

Também deve ser observado que o estudo de interações ecológicas em qualquer tipo de ecossistema, depende do nível e da situação do conhecimento existente e disponível para uso em ecologia. Conforme abordado por Lepsch (2002) estudos de solo não se desenvolvem com profissionais uma única área, portanto o estudo das interações no ecossistema solo prescindem de uma integração multiprofissional e principalmente de integração de diferentes óticas do conhecimento cientifico.

Enquanto a ciência do solo se dedicou muito mais aos processos de interpretação da fertilidade dos solos e suas influencias na produtividade agrícola, a ecologia se ocupou primeiro de conhecer melhor as interações que ocorriam acima do solo. Isso não quer dizer que não se reconhecia por parte dos ecólogos a importância ecológica do solo e suas influencias nas interações que ocorriam acima deste, mas sim que há uma maior quantidade de investigações científicas sobre as interações acima do solo do que nas camadas edáficas em si e no subsolo.

Isso pode ser compreendido se considerarmos as questões metodológicas que norteiam as pesquisas em ecologia de ecossistemas, pois, acima do solo, estas interações são geralmente mais visíveis ou seja, mais facilmente mensuráveis, embora haja certamente exceções. O trabalho de McPhee \& Aarssen (2001) faz uma revisão que em muito contribui nessa discussão, onde os autores avaliam diversas metodologias de estudos de interações de plantas abaixo e acima do solo abordando sobre as possibilidades e limitações de cada uma destas, complementada por levantamento bibliográfico e análise crítica de estudos utilizando cada uma dessas 
metodologias, os autores concluem que todas as metodologias estudadas apresentam aspectos positivos e negativos em relação ao estudo de interações em cada um dos níveis ecossitêmicos (abaixo e acima do solo), e que a principal dificuldade na compreensão integrada das interações above-below ground se deve ao fato de que os métodos de pesquisa geralmente subdividem o ecossistema em: abaixo do solo, e acima do solo, não investigando na maioria das vezes as interações multitróficas que existem ao se considerar os processos de maneira integrada.

$\mathrm{Na}$ teoria essa proposta de integração é muito pertinente, fato que deveria ocorrer ao menos com mais frequência nas pesquisas científicas, pois é provavelmente impossível medir todas as interações que ocorrem com uma única espécie abaixo e acima do solo, mas existe sim a possibilidade de integração de conhecimentos advindos de outros estudos colaborativos a compreensão da interação mútua entre o que ocorre com a espécie abaixo e acima do solo na consideração de estudos multidisciplinares.

De fato a questão teórico-metodológica parece ser um dos principais entraves da análise integrada das interações abaixo e acima do solo, pois nos estudos acima do solo encontra-se atualmente fortes discussões acerca de conceitos como nicho, comunidade, espécie entre outros. Já nos trabalhos que abordam interações abaixo do solo, embora essas discussões teóricas supracitadas também influenciem na ecologia do que acontece abaixo do solo, os problemas mais citados pela maior parte dos pesquisadores como sendo a principal dificuldade da pesquisa no ecossistema solo e subsolo, são sempre relacionadas as metodologias, que não atendem a todos os grupos de organismos presentes no solo, que não identificam associações entre esses organismos a nível inter e intraespecífico e que não são sempre adaptáveis ou mesmo acessíveis para diferentes ecossistemas.

No Brasil, o número de trabalhos sobre os organismos do solo é irrelevante diante da diversidade de ecossistemas do país e do desconhecimento da própria biodiversidade do solo (Souto et al, 2008). No debate sobre a conservação dos recursos naturais, o solo nem sempre tem a atenção necessária para que as ações de conservação sejam efetivas. Por outro lado a degradação dos solos está de certo modo na base de todo o problema da perda cada vez maior de biodiversidade no Brasil. Portanto esse trabalho tem o objetivo de fazer uma revisão do estado da arte no conhecimento sobre as interações ecológicas que ocorrem abaixo e acima do solo de uma perspectiva integrada, avaliando o esforço da pesquisa, as temáticas 
abordadas, as contribuições desses trabalhos para a ecologia de comunidades de organismos de solo e as perspectivas atuais e futuras para essas pesquisas sobre as "above below-ground interactions".

\section{DESENVOLVIMENTO}

\subsection{O estado da arte na pesquisa sobre as interações abaixo e acima do solo de forma integrada: "above-below ground interactions"}

Em uma busca na página eletrônica do Google Acadêmico com o tema: organismos do solo, avaliando-se uma dezena de trabalhos, 7 destes falavam da dificuldade metodológica em se coletar e identificar os organismos que vivem e se interagem superficialmente e abaixo do solo, demonstrando a importância e a necessidade de novos estudos na área de taxonomia e sistemática dos grupos que vivem no ecossistema edáfico, tanto no que se refere a toda a fauna do solo quanto a microflora (GIRACCA et al, 2003; ZILLI et al, 2003; MOÇO et al, 2005; ANTONIOLLI et al, 2006; BARETTA et al, 2008; ROVEDDER et al, 2009; VAZ DE MELO et al, 2009).

Em um levantamento na Revista Brasileira de Ciência do Solo (RBCS) (Fig.1) em sua versão online na página do Scielo Brasil, que se considera uma das mais qualificadas fontes de difusão acadêmica do conhecimento científico acerca dos processos de interação na superfície e abaixo do solo, percebe-se uma grande variação na quantidade de publicações sobre interações ecológicas no ecossistema solo entre o período de 2003 a 2014, o que provavelmente tem a ver com o momento da pesquisa cientifica em cada época e as demandas da sociedade que influenciam nos órgãos e agências de fomento que elaboram editais para financiamento de projetos de pesquisa científica.

Neste levantamento, que considerou apenas a seção de biologia do solo, e os trabalhos realizados em ecossistemas naturais ou em experimentos com espécies nativas foram encontrados 32 publicações, sendo os anos que apresentaram maior quantidade de publicações 2004, 2008 e 2010, em alguns anos como de 2005 a 2007 houve até uma queda na quantidade de publicações, o que mostra que não existe no Brasil um esforço contínuo na produção de conhecimento científico sobre as interações no solo. Já no período de 2011 a 2014 não foi encontrado nenhum trabalho, isso porque a própria seção de biologia do solo praticamente deixou de 
existir, assim como muitas outras seções na revista que relacionavam-se ás demais diversas áreas temáticas da ciência do solo, pois, todos os trabalhos foram nesse período enquadrados nas seções de processos do solo no espaço e no tempo, e na área de manejo do solo que engloba a maior parte das publicações atualmente.

De fato, o momento atual da ciência do solo é o das técnicas de manejo, da diversificação de sistemas, do desenvolvimento de novas formas de aportar matéria orgânica no solo, o que é fomentado sobretudo pela intensa necessidade do aporte de carbono no solo (PROCHNOW, 2013; MOUTINHO, 2014).

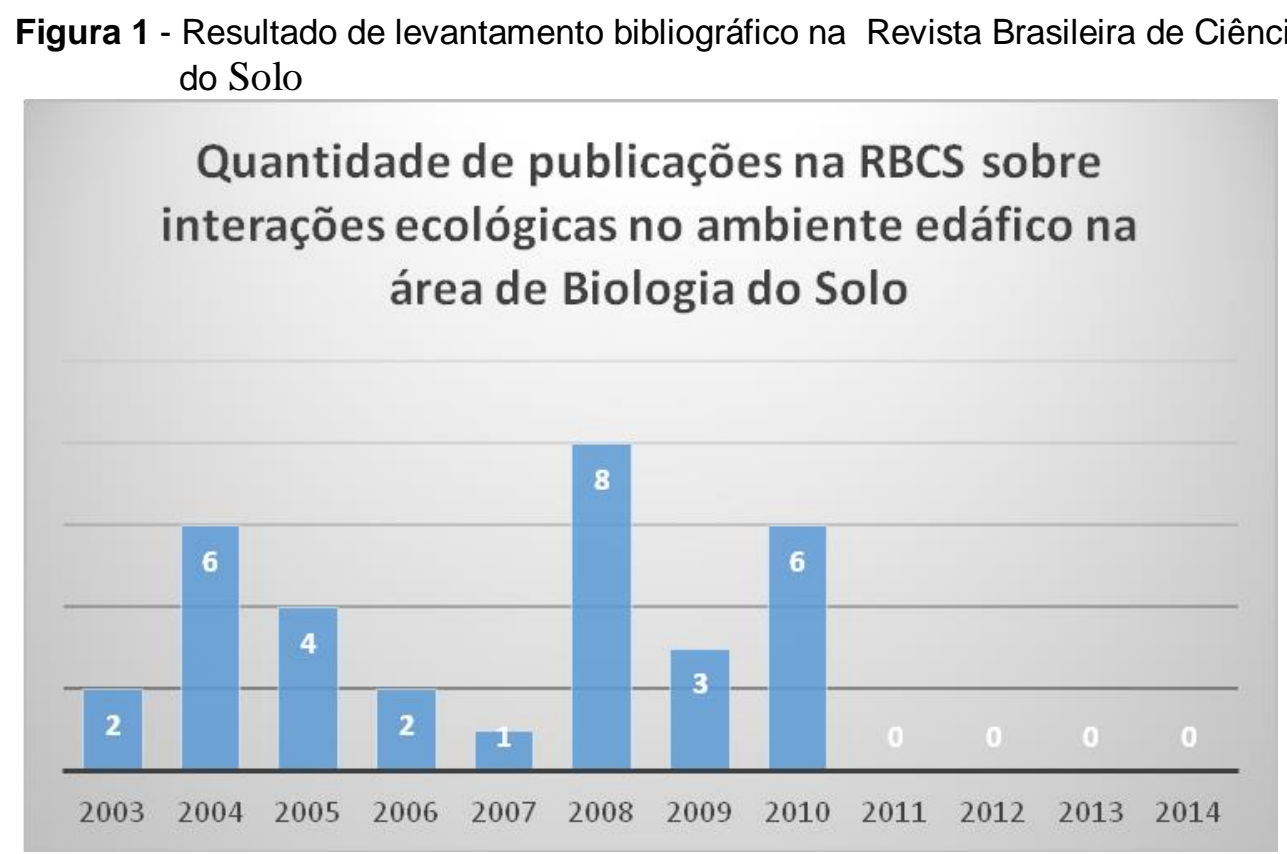

Mas é importante destacar que, muitos dos trabalhos publicados em outras áreas que não a biologia do solo podem trazer contribuições importantes sobre o conhecimento das interações ecológicas no solo, além disso estudos experimentais mesmo os realizados com plantas exóticas cultivadas apesar de haverem muitas limitações na transposição de um conhecimento estritamente agrícola para os ecossistemas naturais podem fornecer importantes informações sobre os processos no sistema solo-planta, sobretudo no que se refere a fertilidade de solos e nutrição de plantas, e com as interações que ocorrem no ecossistema acima do solo como produtividade das plantas em biomassa e defesas. Um outro aspecto a ser considerado é de que como a ciência do solo historicamente está vinculada a produção agrícola, tanto os conceitos dessa ciência quanto suas pesquisas, hipóteses e experimentos 
na maioria das vezes não objetivam conhecer sobre o funcionamento do solo enquanto um ecossistema, mas sim como um agrossistema.

Isso tem melhorado muito nos últimos anos, porque a grande demanda da agricultura de baixo carbono tem como base essencial para o seu desenvolvimento um maior aporte de matéria orgânica no solo (Vezzani \& Mielniczuk, 2009) que por sua vez permite uma complexificação ambiental do ecossistema no solo e consequentemente irá ocasionar uma maior quantidade de habitat proporcionado maior diversidade e riqueza de organismos, o que vai por sua vez influenciar direta ou indiretamente as interações entre as espécies e o solo. Essas melhorias podem ser percebidas no fato de que mesmo o boletim de informações agronômicas do Instituto Internacional de Nutrição de Plantas, cujas publicações são geralmente relacionadas com propostas de utilização de fertilizantes minerais na agricultura brasileira, traz atualmente (CASARIN, 2014) a indicação do livro de Moreira (et al, 2013) como sugestão de boa leitura, destacando-se que este livro aborda sobre os componentes, relações ecológicas e efeitos das interações abaixo e acima do solo na produção vegetal.

Uma das maiores instituições de pesquisa no Brasil na área de solos é a Empresa Brasileira de Pesquisa Agropecuária (EMBRAPA), em uma busca no site desta instituição do período de 2010 a 2014 com o tema interações ecológicas abaixo e acima do solo encontra-se 12.656 resultados de publicações, e com o tema "abovebellow ground interactions" encontra-se 80 resultados que incluem todo o tipo de publicações desde resumos a trabalhos completos de anais de eventos, livros, cartiIhas e boletins técnico científicos.

Em uma análise da quantidade de publicações ao longo dos últimos anos no período de 2001 a 2014 nota-se um crescimento significativo no número de resultados, o que demonstra ter aumentado por parte da Embrapa o esforço da pesquisa sobre a atividade biológica no solo, considerando que a instituição possui a maior parte de suas pesquisas destinadas a produzir conhecimento aplicável para o setor agropecuário brasileiro, isso significa alguns avanços na sustentabilidade ambiental dos ecossistemas do país desde que este conhecimento publicado esteja chegando ao setor produtivo da agropecuária. 
Figura 2 - Resultado de levantamento bibliográfico no site da EMBRAPA

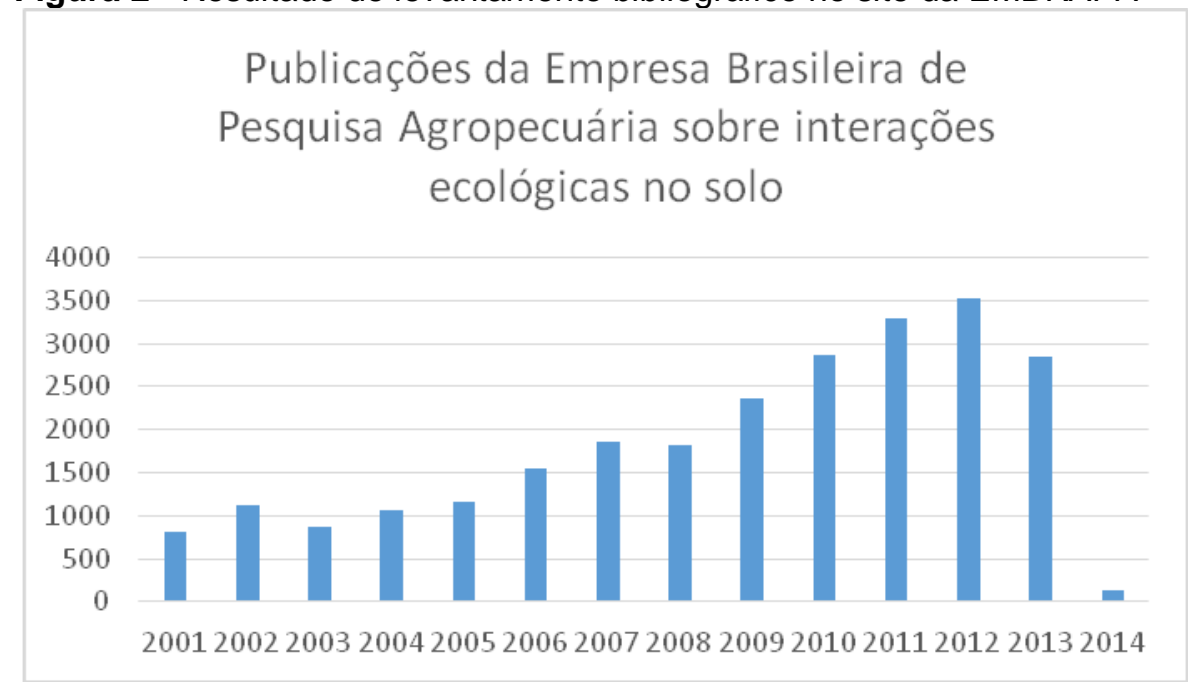

Mas atualmente almeja a construção de um conhecimento ecológico acerca das interações no ecossistema solo, tanto abaixo quanto acima da camada superficial, então é preciso que os ecólogos, se esforcem para o desenvolvimento de mais estudos e pesquisas nesse sentido. Já existem importantes contribuições, na literatura mundial, os estudos sobre os processos de interação abaixo e acima do solo intensificaram-se nas últimas três décadas aproximadamente, o que influenciou muito nas pesquisas brasileiras sobre estas interações.

O trabalho de Singh (et al, 1989) fala da influência das interações abaixo e acima do solo em cultivos agrícolas no semiárido indiano. Mais de vinte anos após esta publicação, Vasconcelos (et al, 2012) trazem uma discussão muito parecida na revista Agropecuária Científica no Semiárido, abordando sobre como as interações no solo podem contribuir para o desenvolvimento da agricultura de pequeno porte no semiárido brasileiro.

Uma das mais extensas publicações sobre os processos de interação abaixo e acima do solo é o livro editado por Persson (1990) que condensa quase trezentas páginas com resultados de pesquisas sobre a inter-relação entre as interações abaixo e acima do solo em ambientes com solos acidificados.

Blomqvist (et al, 2000) estudaram as inter-relações entre interações abaixo e acima do solo em um ambiente de pastagem, e concluíram que as interações abaixo do solo formam micro hábitats na paisagem que correspondem aos mosaicos na vegetação. Wackers \& Bezemer (2003) verificaram que a herbivoria na raiz da planta pode levá-la ao desenvolvimento de defesas indiretas como a produção de néctar extrafloral, mostrando que os herbívoros que se alimentam de raízes podem alterar 
defesas das plantas acima do solo. Soler (et al, 2005) podem ser citados como uma importante contribuição sobre o estudo das interações abaixo e acima do solo de maneira integrada, englobando relações multitróficas, pois estes autores avaliaram se a herbivoria no sistema radicular influencia no desempenho da planta, dos parasitoides, hiperparasitóides e seus inimigos naturais, encontrando resultados positivos que comprovaram que a interação entre o inseto herbívoro e o sistema radicular da planta atuavam no crescimento desta e controle populacional dos organismos associados a espécie acima do solo. Isto sublinha a importância de integrar uma ampla gama de organismos acima e abaixo do solo a fim de facilitar uma melhor compreensão das interações multitróficas complexas e inter-relações entre o que acontece abaixo e acima do solo.

Após quase duas décadas de acúmulo de conhecimentos Wardle (2006) conclui que são as interações bióticas que regulam a biodiversidade no solo. Isso é amplamente reconhecido na maior parte destes trabalhos já citados e nos estudos que foram levantados na RBCS, todavia, é importante destacar a importância dos fatores abióticos que são muitas vezes determinantes nas condições ambientais que irão induzir as espécies a interagirem (FIGUEIREDO et al, 2008), e que portanto não se pode apontar singularmente fatores bióticos ou abióticos como mecanismo principal na manutenção da biodiversidade do solo. O trabalho de Fukami (et al, 2006) aborda sobre o efeito em cascata da introdução de espécies exóticas na diminuição da fertilidade do solo e suas influências no crescimento e desenvolvimento da vegetação. Os trabalhos atuais têm abordado estudos com interações mais complexas envolvendo mais espécies, fatores ambientais e níveis tróficos, nesse contexto Heil (2011) discute que além dos vários fatores não mensuráveis nas inter-relações entre interação abaixo e acima do solo, há muitos processos evolutivos atuando com intensidades desconhecidas em cada organismo envolvido na interação, e que o avanços nos estudos de fisiologia das espécies associados aos de filogenética são necessários para amenizar incongruências entre $o$ que podemos e o que não podemos medir dessas interações abaixo e acima do solo.

Pizano (et al, 2011) avaliaram a influência das interações acima e abaixo do solo atuando de maneira integrada na preferência de algumas espécies arbóreas por diferentes tipos de hábitats. Stevnbak (et al, 2012) relacionou a correlação entre as interações acima e abaixo do solo com os efeitos das mudanças climáticas. Born (et al, 2013) fez um trabalho muito parecido com o de Pizano (et al, 2011), onde avaliou 
a interação entre diferentes espécies de um mesmo gênero entre plantas especialistas e generalistas com relação ao solo com diferentes fatores abióticos como saturação hídrica, disponibilidade de luz e variações pedogeomorfológicas ao longo do desenvolvimento vital dessas plantas, constatando que as espécies possuem adaptações a determinados ambientes que foram adquiridas ao longo da história de vida da espécie.

$\mathrm{Na}$ literatura brasileira como exemplo de publicação que condensa vários trabalhos sobre a interação abaixo e acima do solo podemos citar o livro editado por Figueiredo (et al, 2008) que aborda enfaticamente a ação dos micro-organismos do solo para promoção de uma agricultura mais sustentável e uma maior compreensão dos processos ecológicos sobre a biodiversidade existente no solo com 24 capítulos abordando diferentes assuntos como a fixação biológica de nitrogênio, a influência dos micro-organismos no crescimento das plantas, componentes orgânicos do solo, biofertilizantes e recuperação de áreas degradadas, trazendo muitos resultados de pesquisas com espécies nativas tanto de plantas quanto de micro-organismos como bactérias fixadoras.

Nos trabalhos brasileiros as pesquisas sobre as interações abaixo do solo se concentram no estudo de micro-organismos, pois a maioria dos 32 trabalhos avaliados da RBCS abordavam interações abaixo e ou acima do solo a partir de estudos com micro-organismos (SILVA et al, 2010; GIONGO et al, 2010; MARTINS ET AL, 2010), principalmente a biomassa microbiana que tem sido o principal componente utilizado para avaliar a qualidade e a atividade biológica do solo (ZILLI et al, 2003), isso devido a grande evolução científica pela qual tem passado os estudos com marcadores moleculares (FIGUEIREDO et al, 2008; GOULART et al, 2011) que são determinantes na identificação filogenética e mesmo taxonômica dos organismos presentes na biomassa microbiana e também na fauna do solo, e também pela maior sensibilidade desses organismos as alterações ocorridas no ambiente ao longo do espaço e do tempo.

Os estudos com organismos do solo da macro e meso fauna geralmente trabalham com levantamento de espécies avaliando riqueza, abundância, diversidade e características funcionais e a possibilidade desses organismos servirem como indicadores de parâmetros físico-químicos de solo como nível de aeração, estruturação de agregados e fertilidade (Vaz de Melo et al, 2009). 
Um trabalho com abordagem mais integrada das interações abaixo e acima do solo é o de Baretta (et al, 2008) que aborda sobre a interação de um grupo organismos do solo com espécies arbóreas dominantes em seu hábitat natural, concluindo que a pressão antrópica sobre a planta que tem diminuído suas populações tem ocasionado também uma diminuição na biodiversidade de organismos da fauna edáfica e biomassa microbiana associadas a espécie.

Rovedder (et al, 2009) fizeram um trabalho avaliando a eficácia da utilização de plantas nativas na recuperação de solos degradados por arenização, e constataram que os organismos do solo possuem interações tróficas mais fortes com as espécies nativas e que a grande utilização de plantas exóticas na recuperação de áreas degradadas muitas vezes pode alterar a atividade biológica dos organismos do solo, porque as plantas nativas atuam na modificação da estrutura física e química do solo em uma intensidade e funcionalidade em que os organismos do solo conseguem acompanhar e se adaptar evolutiva e comportamentalmente a essas mudanças.

Um assunto a parte na ciência sobre as interações abaixo e acima do solo de forma inter-relacionada são os ambientes cavernícolas que atuam como reguladores tanto na dinâmica abiótica do ecossistema servindo como reservatórios hidrológicos, e também como parte do hábitat de sobrevivência de algumas populações e comunidades de espécies que necessitam tanto da caverna como do ecossistema superficial para viverem (Cavalcanti et al, 2012). No Brasil, o congresso brasileiro de espeleologia que acontece a cada dois anos tem publicado muitos trabalhos sobre as interações ecológicas que envolvem os organismos cavernícolas e o ambiente superficial, foi feito o levantamento nos anais de trabalhos completos do $30^{\circ}, 31^{\circ}$ e $32^{\circ}$ congresso brasileiro de espeleologia de pesquisas envolvendo as interações abaixo e acima do solo em ambientes com ocorrência de cavidades naturais subterrâneas, os resultados mostram que foram publicados 34 trabalhos na área de bioespeleologia entre os anos de 2009 a 2013.

\section{CONCLUSÕES}

No contexto atual da conservação de recursos naturais, não é possível que os estudos tanto em ecologia quanto na ciência do solo possam evoluir num caminho à negligenciar os aspectos da biologia do solo, das interações ecológicas naturais en- 
tre plantas, herbívoros e seus inimigos naturais e todos os processos associados as interações abaixo e acima deste ecossistema. Ocorre uma lacuna que precisa ser preenchida com o melhor conhecimento do solo, saber quem vive e como vive nele, porque e a que custo esses organismos se adaptaram a viver nesse ecossistema. Demandas para pesquisas nessa área são muitas, não há como se pensar a conservação de maneira holística se todas as ciências não se dispuserem a colaborar com a tarefa de dar suas contribuições na produção de conhecimento sobre os processos de inter-relação que regem o equilíbrio sistemático da biodiversidade, sendo o solo a base e meio principal de todo esse equilíbrio, portanto precisa ser cada vez mais estudado e aprimorados seus métodos de analise de estudo, onde forneçam dados mais confiáveis, reprodutivos que geram maior multidisciplinaridade entre as ciências envolvidas nos estudos dos ecossistemas .

\section{AGRADECIMENTOS}

Gostaria de agradecer a professora Leidivan Almeida Frazão pela grande ajuda com a indicação de referências bibliográficas, a CAPES pela bolsa de pesquisa e a todos do Laboratório de Ecologia e Propagação Vegetal pela ajuda com as discussões.

\section{REFERÊNCIAS}

ANTONIOLLI, Z.I.; CONCEIÇÃO, P.C.; BÖCK, V.; PORT, O.; SILVA, D.M.; SILVA, R.F. Método alternativo para estudar a fauna do solo. Revista Ciência Florestal, Santa Maria, v. 16, n. 4, 2006.

BARETTA, D.; FERREIRA, C.S.; SOUSA, J.P.; CARDOSO, E.J.B.N. Colêmbolos (Hexapoda: Collembola) como bioindicadores de qualidade do solo em áreas com Araucaria angustifólia. Revista Brasileira de Ciência do Solo, v.32, número especial, 2008.

BLOMQVIST, M. M.; OLFF, H.; BLAAUW, M. B.; BONGERS, T.; VAN DER PUTTEN, W. H. Interactions between above- and belowground biota: importance for small-scale vegetation mosaics in a grassland ecosystem. Oikos, n.90, Copenhague, 2000.

BORN, J.; PLUES, A.R.; BURSLEM, D.F.R.P.; NILU, R.; MAYCOC, C.R.; GHAZOUL, J. Differing Life History Characteristics Support Coexistence of Tree Soil Generalist and Specialist Species in Tropical Rain Forests. Biotropica, 2013.

CAVALCANTI, L.F.; LIMA, M.F.; MEDEIROS, R.C.S.; MEGUERDITCHIAN, I. (Orgs.) Plano de ação nacional para a conservação do patrimônio espeleológico nas áreas carsticas da Bacia do rio São Francisco. Série Espécies Ameaçadas nํ27, ICMBIO, CECAV, Brasília, 2012. 
DENARDIN, J. E.; FAGANELLO, A.; DENARDIN, N. D.; KOCHHANN, R. A. Fertilidade do solo: conhecimentos, aplicações, interfaces e desafios. SIMPÓSIO MINEIRO DE CIÊNCIA DO SOLO, 2., 2013. Anais... Viçosa, 2013.

FUGUEIREDO, M.V.B.; BURITY, H.A.; STAMFORD, N.P.; SANTOS, C.E.R.S. Microorganismos e agrobiodiversidade: o novo desafio para agricultura. Guaíba: Agrolivros, 2008.

FUGUEIREDO, M.V.B.; LIRA-JUNIOR, M.A.; ARAÚJO, A.S.F.; MARTINEZ, C.R. Fatores bióticos e abióticos à fixação biológica de $\mathrm{N}_{2}$. In: FUGUEIREDO, M.V.B.; BURITY, H.A.; STAMFORD, N.P.; SANTOS, C.E.R.S. Microorganismos e agrobiodiversidade: o novo desafio para agricultura. Guaíba: Agrolivros, 2008.

FUKAMI, T.; WARDLE, D.A.; BELLINGHAM, P.J.; MULDER, C.P.H.; TOWNS, D.R.; YEATES, G.W.; BONNER, K.I.; DURRETT, M.S. GRANT-HOFFMAN, M.N.; WILLIAMSON, W.M. Above- and below-ground impacts of introduced predators in seabird-dominated island ecosystems. Ecology Letters, n.9, 2006.

GIONGO, A.; BENEDUZI, A.; AMBROSINI, A.; VARGAS, L.K.; STROSCHEIN, M.R.; ELTZ, F.L.; BODANESE-ZANETTINI, M.H.; PASSAGLIA, L.M.P. Isolation and characteration of two plant growth-promoting bactéria from the rhizoplane of a legume (Lupinus albescens) in sandy soil. Revista Brasileira de Ciência do Solo, v.34, 2010.

GIRACCA, E.M. N.; ANTONIOLLI, Z. I.; ELTZ, F. L. F.; BENEDETTI, E.; LASTA, E.; VENTURINI, S. F.; VENTURINI, E. F.; BENEDETTI, T. Levantamento da meso e macrofauna do solo na microbacia de Arroio Lino, Agudo/RS. Revista Brasileira de Agrociência, v. 9, n. 3, 2003.

GOULART, I.C.G.R.; MEROTTO JUNIOR, A.; NUNES, A.L.; BERED, F. Otimização da utilização de marcadores moleculares microssatélites e sua aplicação em estudos com plantas daninhas. Revista Planta Daninha, v.29, número especial, Viçosa, 2011.

Heil, M. Plant-mediated interactions between above- and below-ground communities at multiple trophic levels. Journal of Ecology, n. 99, 2011.

LEPSCH, I.F. Formação e conservação dos solos. Ed. Oficina de Textos, São Paulo, 2002.

MARTINS, C.M.; GALINDO, I.C.L.; SOUZA, E.R.S; POROCA, H.A. Atributos químicos e microbianos do solo de áreas em processo de desertificação no semiárido de Pernambuco. Revista Brasileira de Ciência do Solo, v.34, 2010.

MCPHEE, C.S.; AARSSEN, L.W. The separation of above and below-ground competition in plants - A review and critique of methodology. Plant Ecology, v.152, p.119-136, 2001.

MOÇO, M.K.S.; GAMA-RODRIGUES, E.F.; GAMA-RODRIGUES, A.C.; CORREIA, M.E.F. Caracterização da fauna edáfica em diferentes coberturas vegetais na região norte fluminense. Revista Brasileira de Ciência do Solo, v. 29, p.555-564, 2005.

MOUTINHO, P. Desmatamento na Amazônia: desafios para reduzir as emissões de gases de efeito estufa do Brasil. Fundação Brasileira para o Desenvolvimento Sustentável. Acessado em 29 de maio de 2014.

PERSSON, H. Above and below-ground interactions in forest trees in acidified soils. Relatório 32 de Pesquisas sobre a Poluição do Ar. Swedish University of Agricultural Sciences Department of Ecology and Environmental Research, Uppsala, 1990. 
PIZANO, C.; MANGAN, S.A.; HERRE, E.A.; EOM, A.; DALLING, J.W. Above- and belowground interactions drive habitat segregation between two cryptic species of tropical trees. Ecology, v. 92, n.1, 2011.

ROVEDDER, A.P.M.; ELTZ, F.L.F.; DRESCHER, M. S.; SCHENATO, R. B.; ANTONIOLLI, Z.I. Organismos edáficos como bioindicadores da recuperação de solos degradados por arenização no Bioma Pampa. Revista Ciência Rural, v.39, n.4, 2009.

SINGH, R.P.; ONG, C.K.; SAHARAN, N. Above and below ground interactions in alleycropping in semi-arid India. Agroforestry Systems, n.9, 1989.

SILVA, R.R.; SILVA, M.L.N.; CARDOSO, E.L.; MOREIRA, F.M.S.; CURI, N. ALOVISI, A.M.T. Biomassa e atividade microbiana em solo sob diferentes sistemas de manejo na região fisiográfica campo das vertentes - MG. Revista Brasileira de Ciência do Solo, v.34, 2010.

SOLER, R.; BEZEMER, T.M.; PUTTEN, W.H.V.B.; VET, L.E.M.; HARVEY, J.A. Root herbivore effects on above-ground herbivore, parasitoid and hyperparasitoid performance via changes in plant quality. Journal of Animal Ecology, n.74, 2005.

SOUTO, P.C.; SOUTO, J.S.; MIRANDA, J.R.P.; SANTOS, R.V.; ALVES, A.R. C. Comunidade microbiana e mesofauna edáficas em solo sob caatinga no semiárido da Paraíba. Revista Brasileira de Ciência do Solo, v.32, n. 32, Viçosa, 2008.

STEVNBAK, K.; SCHERBER, C.; GLADBACH, D.J.; BEIER, C.; MIKKELSEN, T.N.; CHRISTENSEN, S. Interactions between above- and belowground organisms modified in climate change experiments. Nature Climatic Change, 2012.

VASCONCELOS, M.C.C.A.; SILVA, A.F.A.; LIMA, R.S. Cultivo em aléias: uma alternativa para pequenos agricultores. Revista Agropecuária Científica no Semiárido, v. 8, n.3, 2012.

VAZ-DE-MELO, F.; BROWN, G.G.; CONSTANTINO, R.; LOUZADA, J.N.C.; LUIZÃO, F.J.; MORAIS, J.W.; ZANETTI, R. A importância da meso e macrofauna do solo na fertilidade e como bioindicadores. Boletim Informativo da Sociedade Brasileira de Ciência do Solo, Seção de Biologia do Solo, 2009.

VEZZANI, F.M.; MIELNICZUK, J. Uma visão sobre qualidade do solo. Revista Brasileira de Ciência do Solo, v.33, 2009.

WACKERS, F.; BEZEMER, T.M. Root herbivory induces an above-ground indirect defence. Ecology Letters, n.6, 2003.

WARDLE, D.A. The influence of biotic interactions on soil biodiversity. Ecology Letters, v.9, 2006.

ZILLI, J.E RUMJANEK, N.G.; XAVIER, G.R.; COUTINHO, H.L.C.; NEVES, M.C.P. Diversidade microbiana como indicador de qualidade de solo. Cadernos de Ciência \& Tecnologia, Brasília, v. 20, n. 3, 2003. 